\title{
Increasing Trend and Characteristics of Korean Adolescents Presenting to Emergency Department for Self-Harm: A 5-Year Experience, 2015 to 2019
}

\author{
Jooah Cheon, Donghun Oh, Junghan Lee, Jaeun Ahn, Dong-Ho Song, and Keun-Ah Cheon \\ Division of Child and Adolescent Psychiatry, Department of Psychiatry, Severance Hospital, Institute of Behavioral Science in Medicine, \\ Yonsei University College of Medicine, Seoul, Korea.
}

\begin{abstract}
Purpose: Emergency department (ED) is a common treatment setting for adolescents with clinically serious self-harm. Here, we investigated the clinical characteristics and trends of adolescents with self-harm who visited the ED in one Korean university hospital. We also compared patients with a single ED visit to those with multiple ED visits to identify the risk factor of repeated visits. Materials and Methods: We retrospectively identified patients aged 12 to 18 years who presented to ED for self-harm from January 2015 to December 2019, based on electronic medical records. Self-harm included all thoughts and behaviors indicating intents to harm or hurt oneself, regardless of the degree of such attempt.

Results: A total of 168 individuals (male:female=31:137; average 15.99 \pm 1.64 years) presented to ED following 304 episodes (45 and 259 episodes in males and females, respectively). The number of episodes steeply increased between 2016 and 2019, and the overall number during the study showed an increasing trend $(p=0.043)$. Repeated ED visitors with self-harm showed more history of psychiatric treatment/admission ( $58.3 \%$ vs. $85.4 \%, p=0.002 ; 14.2 \%$ vs. $43.9 \%, p<0.001)$, history of child abuse (32.3\% vs. $53.7 \%$, $p=0.013)$, and familial psychiatric history ( $13.4 \%$ vs. $31.7 \%, p=0.008)$ compared to those with a single visit.

Conclusion: Among Korean adolescents, the number of ED visits and repetition of ED visits for self-harm is on the rise. For adolescents presenting to ED with self-harm, the history of psychiatric treatment/admission, child abuse, and familial psychiatric history should be properly obtained to identify the risk for multiple ED visits.
\end{abstract}

Key Words: Adolescent, self-injurious behavior, emergency departments

\section{INTRODUCTION}

Suicide in adolescents is a major public health problem and

Received: February 13, 2020 Revised: April 24, 2020

Accepted: May 18, 2020

Corresponding author: Keun-Ah Cheon, MD, PhD, Division of Child and Adolescent Psychiatry, Department of Psychiatry, Severance Hospital, Institute of Behavioral Science in Medicine, Yonsei University College of Medicine, 50-1 Yonsei-ro, Seodaemun-gu, Seoul 03722, Korea.

Tel: 82-2-2228-1633, Fax: 82-2-313-0891, E-mail: KACHEON@yuhs.ac

-The authors have no potential conflicts of interest to disclose.

(C) Copyright: Yonsei University College of Medicine 2020

This is an Open Access article distributed under the terms of the Creative Commons Attribution Non-Commercial License (https://creativecommons.org/licenses/ by-nc/4.0) which permits unrestricted non-commercial use, distribution, and reproduction in any medium, provided the original work is properly cited. one of the leading causes of death worldwide. ${ }^{1}$ In Korea, suicide has been the most common cause of death in adolescents since 2007. ${ }^{2}$ Among various risk factors of suicide, nonfatal self-harm behavior is a significant and growing public health concern. A recent meta-analysis estimated that one in 25 patients presenting to hospital for self-harm will die by suicide within the next 5 years. ${ }^{3}$ Suicide rate and self-harm behavior are rapidly increasing in adolescents, especially among females. ${ }^{4}$ For this reason, adolescents who visit the hospital after self-harm are an important target for suicide prevention. One of the important characteristics of adolescent suicide is its social contagion and modeling on suicide behavior., ${ }^{5,6}$ The majority of studies support positive associations between exposure to peer suicidal behavior and adolescent suicide attempts, and there is mounting evidence of the media's influence on 
suicide-related behavior in adolescents. ${ }^{5}$ Along with suicidal attempts, adolescents with non-suicidal self-injury are also vulnerable to peer and media influences. ${ }^{6,7}$ For adolescents who sustain clinically serious self-harm injuries, the emergency department (ED) is the most common treatment setting, and it was recommended to be prioritized for research and intervention efforts by the National Strategy for Suicide Prevention in the United States. ${ }^{8}$ Although the majority of self-harm incidents in children and adolescents visiting ED were not fatal, these young people have been identified as a high-risk group for suicide attempts, with the greatest risk occurring in the period immediately after the episode of self-harm. ${ }^{8,9}$

Repeated self-harm is common, especially in the first week after an index hospital presentation. ${ }^{10,11}$ Repetition of self-harm is indicative of persistent distress, and it increases the individual and societal costs: compared to those who engage in single episode of self-harm only, those with repeated self-harm are more than twice as likely to die by suicide. ${ }^{12,13}$ Although research has shown changing patterns of risk factors for self-harm and its repetition, the majority of studies examining ED visits for selfharm in adolescents have been conducted in western countries. ${ }^{3,14}$ Recent evidence from Taiwan reported that the risk of repeated self-harm risk within 1 year was $10.5 \%$ in adolescents and young people, which is lower than that in western countries, where among young people, self-harm by medicine overdose is increasing and repeat episodes of self-harm is a risk factor for repetition. ${ }^{15}$ Understanding the factors that influence the rate and pattern of repetition of adolescent self-harm at ED will help to establish the prevention strategies and to optimize follow-up after a self-harm episode.

Therefore, this study investigated the clinical characteristics and trends of adolescents who visited the ED with self-harm in one Korean university hospital from 2015 to 2019. We also compared the patients with single ED visits to those with multiple ED visits to identify the risk factors of repeated visit for self-harm.

\section{MATERIALS AND METHODS}

\section{Study population}

We identified patients aged 12 to 18 years who presented to EDs for self-harm from January 2015 to December 2019, based on electronic medical records of Severance Hospital in Seoul, Korea. In our study, self-harm referred to all thoughts and behaviors about intentional harm to oneself, regardless of the degree of suicidal intent or medical seriousness. Therefore, adolescents with self-harm included all those who visited for suicidal ideation, plan, attempts and gestures, non-suicidal self-injury, and non-suicidal drug overdose. We identified the patients using International Classification of Diseases, 10th edition (2010 version) codes for suicidal ideations [R45.8]; laceration of shoulder and upper arm [S40 to S41]; laceration of elbow and forearm [S50 to S51]; laceration of wrist and hand [S60 to S61]; laceration of hip and thigh [S70 to S71]; laceration of knee and lower leg [S80 to S81]; laceration of ankle and foot [S90 to S91]; poisoning by drugs, medicaments, and biological substances [T36 to T50]; toxic effects of substances chiefly nonmedicinal as to source [T51 to T65]; intentional self-poisoning [X60 to X69]; intentional self-harm by hanging, strangulation, and suffocation [X70]; intentional self-harm by drowning and submersion [X71]; intentional self-harm by steam, hot vapor, and hot objects [X77]; intentional self-harm by sharp object [X78]; intentional self-harm by blunt object [X79]; intentional self-harm by jumping from a high place [X80]; intentional selfharm by jumping or lying in front of a moving object [X81]; intentional self-harm by crashing of motor vehicle [X82]; intentional self-harm by other specified means [X83]; personal history of self-harm [Z91.5]; sequelae of intentional self-harm [Y87.0]; and self-damaging behavior [Z72.8].

We excluded duplicated cases with multiple codes and cases without the intention of self-harm after reviewing all of the available medical records of patients, especially psychiatric consultation notes of the adolescents. We collected the information about gender, age, day of visit, reason for visiting ED, type of methods used for suicide or self-harm, major stressors, prior psychiatric treatments, psychiatric diagnosis, history of child abuse, psychiatric family history, and patient disposition. A patient's first self-harm presentation during the study period was defined as his or her index episode. Methods of selfharm were categorized into self-poisoning, self-cutting, charcoal burning, jumping from the height, hanging, strangulation, and other methods. A key method was defined as the most lethal method used by an individual with multiple ED visits. For example, if a patient visited the ED for self-harm episodes with hanging and self-cutting, hanging was identified as the key method for the patient based on method lethality. This study was approved by the Institutional Review Board of Severance hospital, Yonsei University College of Medicine, and the acquisition of informed consent was waived for this retrospective study (IRB number; 2019-3386-001).

\section{Statistical analysis}

Continuous variables are expressed as the mean and standard deviation or median with range. Continuous variables were compared using independent t-tests, while categorical variables were compared using chi-square test and Fisher's exact tests, as appropriate. Mann-Kendall trend test was used to identify the trend by year and age. Statistical significance was set at $p<0.05$. All statistical analyses were performed using $\mathrm{R}$ software (version 3.3.2, R Foundation for statistical computing, Vienna, Austria, available under open source licensing http:// www.r-project.org/). 


\section{RESULTS}

\section{Patient characteristics}

From January 2015 to December 2019, 168 individuals aged between 12 and 18 years (average $15.99 \pm 1.64$ years) presented to the ED (Table 1) following a total of 304 episodes ( 45 by males and 259 by females). Based on the demographic characteristics of patients at their index episodes, most patients were female $(\mathrm{n}=137,81.6 \%)$, with a female-to-male ratio of $4.42: 1$, and the number of patients was increased according to age (MannKendall trend test; overall, $p=0.001$; female, $p=0.003$; male, $p=$ 0.003 ). The number of 18 -year-old male patients was roughly triple that of other age male patients, showing the least femaleto-male ratio of 2.57:1 at the age of 18 (Fig. 1).

The number of episodes steeply increased between 2016 and 2019, and the overall number during this study showed an increasing trend (Mann-Kendall trend test, $p=0.043$ ) (Fig. 2). This pattern was primarily seen in females. In males, while there was a slightly decreased number of visits in 2018 , probably due to relatively small numbers, the overall trend was increasing during this study. Between 2016-2017 and 2018-2019,

Table 1. Numbers of Persons and Episodes Who Referred to the Emergency Department after Self-Harm, by Year and Sex

\begin{tabular}{|c|c|c|c|c|c|c|}
\hline \multirow{2}{*}{ Year } & \multicolumn{2}{|c|}{ Males } & \multicolumn{2}{|c|}{ Females } & \multicolumn{2}{|c|}{ Both } \\
\hline & Persons & Episodes & Persons & Episodes & Persons & Episodes \\
\hline 2015 & 0 & 0 & 17 & 26 & 17 & 26 \\
\hline 2016 & 3 & 3 & 13 & 21 & 16 & 24 \\
\hline 2017 & 9 & 10 & 21 & 25 & 30 & 35 \\
\hline 2018 & 7 & 9 & 46 & 77 & 53 & 86 \\
\hline 2019 & 12 & 23 & 40 & 110 & 52 & 133 \\
\hline Total & 31 & 45 & 137 & 259 & 168 & 304 \\
\hline
\end{tabular}

there was a $66 \%$ increase in the number of episodes presenting to $\mathrm{ED}$ ( 46 vs. 187). This increase was greater among females (406.5\%; 46 vs. 187 ) than among males (246.2\%; 13 vs. 32 ).

In Fig. 3, there seems to be a monthly variation, with a decrease in the number of episodes in March and August, and even fewer episodes in January and February. However, after taking into account the differing lengths of the month, this trend was not significant (chi-square goodness of fit, $\chi^{2}=9.17, p=0.606$ ).

\section{Clinical characteristic by reason for emergency department visit}

Patients visiting the ED with self-harm episodes were divided into three groups as follows: suicidal ideation only ( $n=76)$, suicidal self-harm $(n=117)$, and non-suicidal self-harm $(n=111)$. Demographics and clinical information are summarized in Table 2. There was no significant difference in sex and age. In suicidal self-harm, self-poisoning was the sole method in $56.4 \%$ of the episodes, jumping from height in $23.1 \%$, self-cutting in $16.3 \%$, hanging or strangulation in $5.2 \%$, and charcoal burning in $2.6 \%$. In non-suicidal self-harm, self-cutting was the sole method in $54.9 \%$ of the episodes, self-poisoning in $46.8 \%$, and hanging or strangulation in $1.8 \%$. Self-cutting was significantly higher in non-suicidal self-harm patients $(p<0.001)$, whereas jumping from height was significantly higher in suicidal selfharm patients $(p<0.001)$. Stabbing, self-biting, and self-hitting were also reported in one episode. History of ED visit and psychiatric admission were significantly higher in the suicidal ideation only group than in the suicidal self-harm and non-suicidal self-harm groups. However, a history of suicidal behavior or psychiatric treatment was not different among groups. More patients in the suicidal self-harm $(56 / 117,47.8 \%)$ and nonsuicidal self-harm groups $(53 / 111,47.8 \%)$ reported a history of child abuse than did patients in the suicidal ideation only

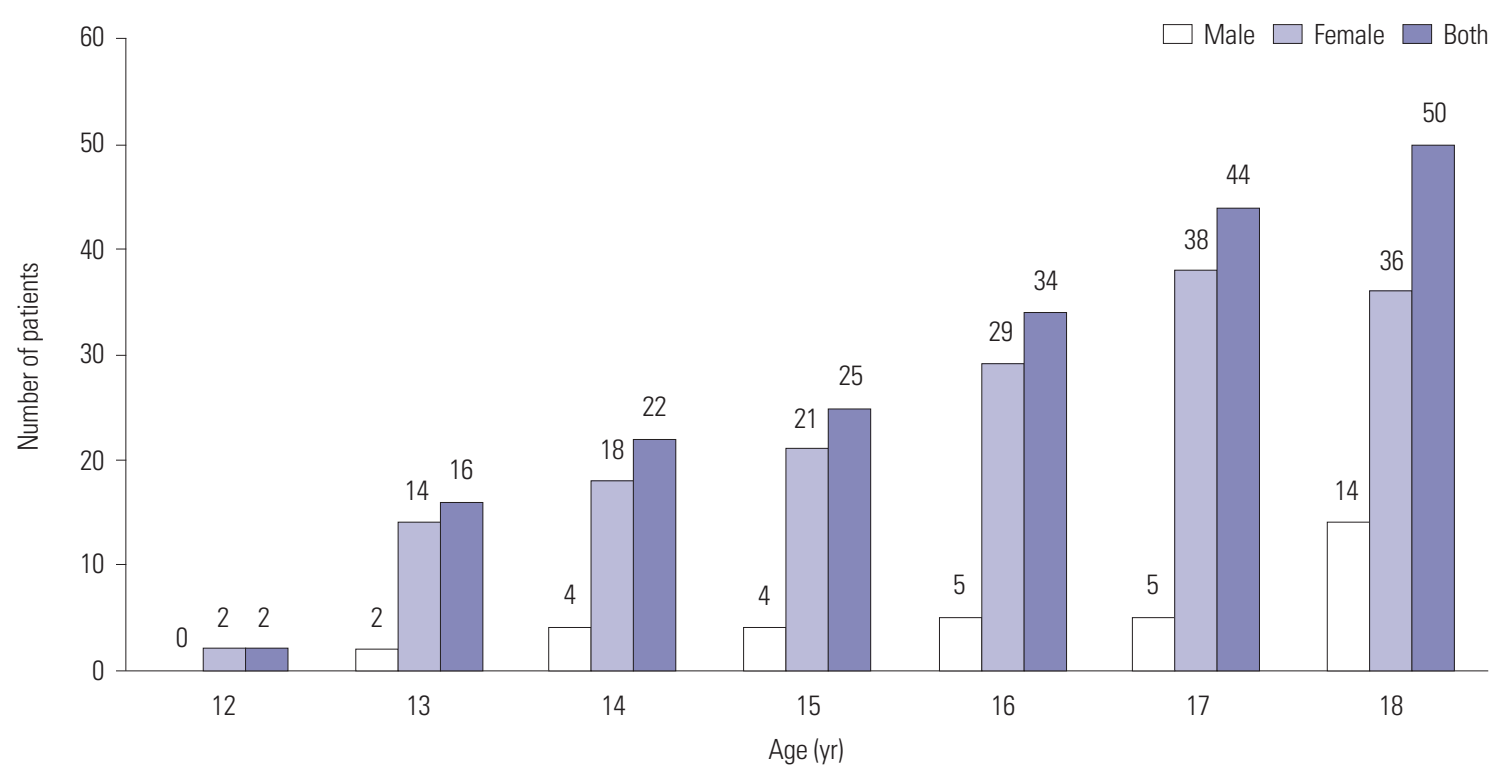

Fig. 1. Number of patients by age at the first ED visit and gender during the observed period. ED, emergency department. 


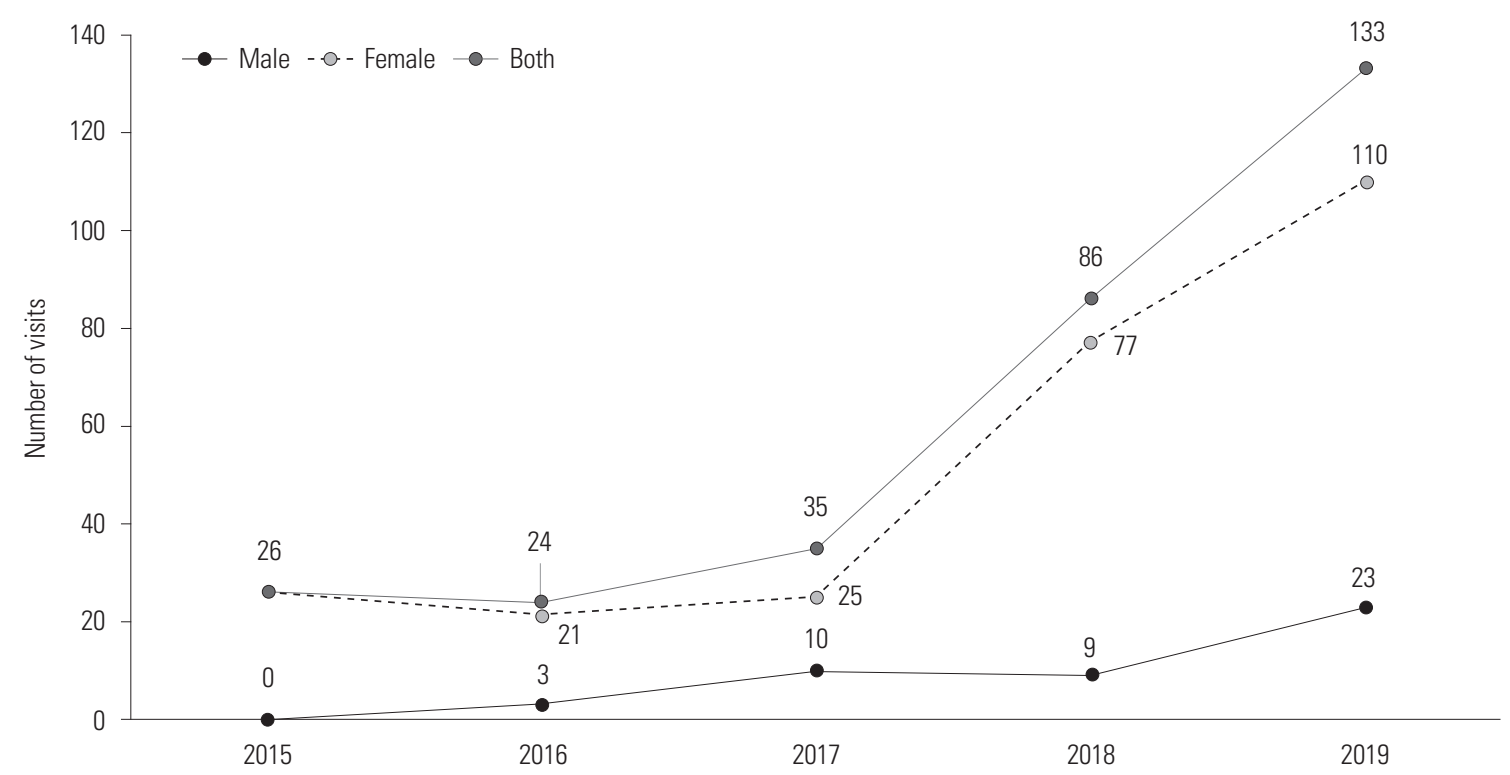

Fig. 2. Trend of number of ED visits in the observation period. ED, emergency department.

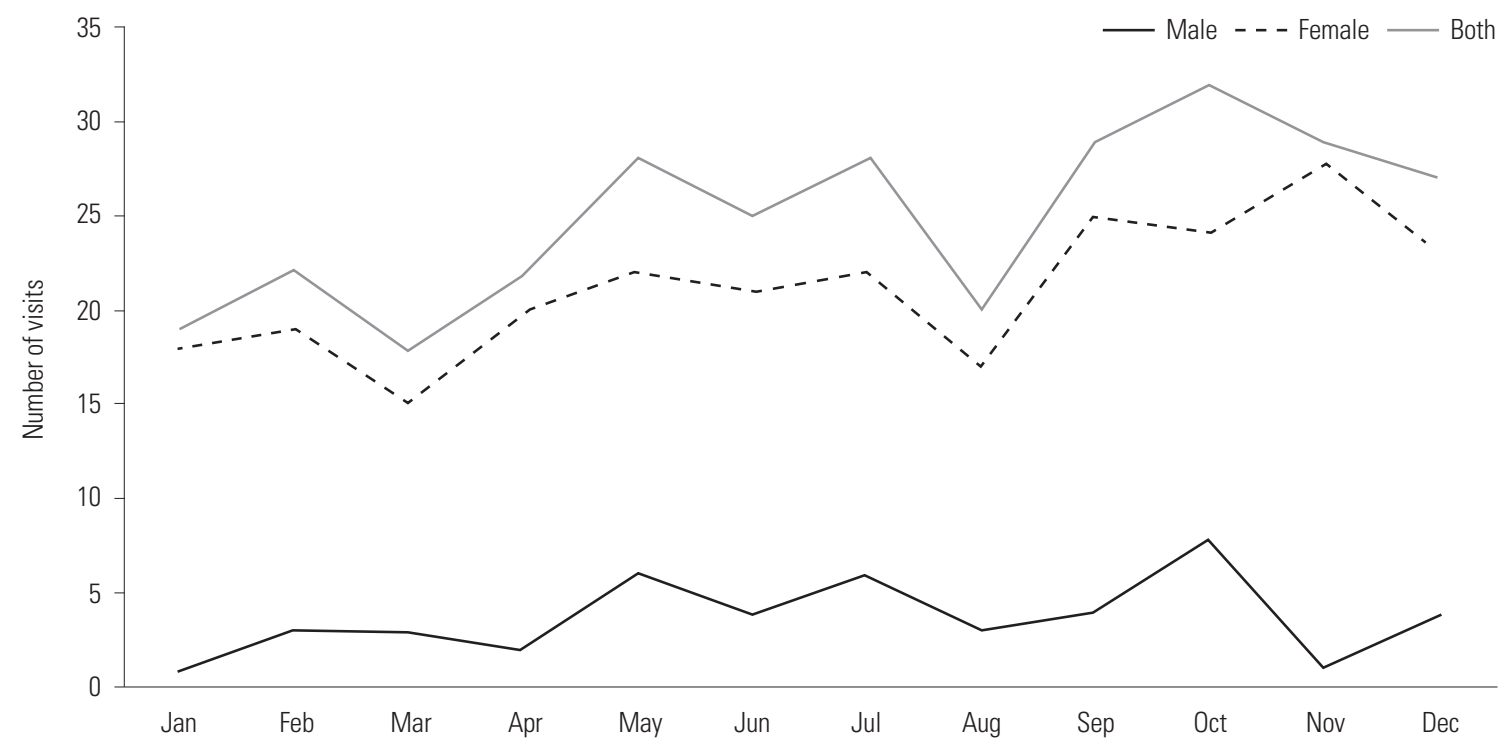

Fig. 3. Number of ED visits by months. ED, emergency department.

group (16/76, 21.1\%; $p<0.001)$. Unknown history of child abuse in the suicidal self-harm, non-suicidal self-harm, and suicidal ideation only groups were reported in 14/117 (12.0\%), 9/111 (8.1\%), and 7/76 (9.2\%), respectively, without statistical difference. Among types of child abuse, emotional and neglect types were significantly higher in suicidal and non-suicidal self-harm groups. For major stressors, relationship with family was the leading cause in the suicidal and non-suicidal self-harm groups, with a significantly higher proportion compared to the ideation only group ( $9.2 \%$ vs. $39.3 \%$ vs. $42.3 \%$; $p<0.001$ for both comparisons). In contrast, no specific stressor was identified in the ideation only group ( $60.5 \%$ vs. $27.3 \%$ vs. $35.1 \%$; $p<0.001$ for both comparisons). Depression was higher in the suicidal and nonsuicidal self-harm groups ( $47.4 \%$ vs. $73.5 \%$ vs. $73.0 \% ; p<0.001$ for both comparisons), whereas bipolar disorder was higher in the ideation only group ( $28.9 \%$ vs. $9.4 \%$ vs. $7.2 \%$; $p<0.001$ for both comparisons). While the rate of admission to psychiatry was lower in the non-suicidal self-harm group, the rate of admission to other departments or transfer was higher in the suicidal self-harm group than in the other two groups. Discharge with follow-up in the outpatient department (OPD) was higher in the ideation only group (64.5\%) than in the suicidal self-harm $(40.2 \%, p<0.001)$ and non-suicidal self-harm groups $(48.6 \%$, $p=0.033$ ). In contrast, discharge without follow-up in the OPD was higher in the non-suicidal self-harm group (38.7\%) than in the suicidal self-harm $(26.5 \%, p=0.048)$ and ideation only groups $(14.5 \%, p<0.001)$. Comorbid diagnosis is summarized in Supplementary Table 1 (only online). 
Table 2. Demographic and Clinical Characteristics of Adolescent Self-Harm Episodes in the ED

\begin{tabular}{|c|c|c|c|}
\hline $\begin{array}{l}\text { Reason for ED visits } \\
\end{array}$ & Suicidal ideation only & Suicidal self-harm & Non-suicidal self-harm \\
\hline Number of episodes & 76 & 117 & 111 \\
\hline Sex, female & $61(80.3)$ & $103(88.0)$ & $95(85.6)$ \\
\hline Age, mean $\pm S D$ & $15.99 \pm 1.64$ & $15.95 \pm 1.65$ & $15.97 \pm 1.64$ \\
\hline \multicolumn{4}{|l|}{ Method* } \\
\hline Self-poisoning & - & $66(56.4)$ & $52(46.8)$ \\
\hline Self-cutting ${ }^{\S}$ & - & $19(16.3)$ & $61(54.9)$ \\
\hline Charcoal burning & - & $3(2.6)$ & $0(0.0)$ \\
\hline Jumping from height ${ }^{\S}$ & - & $27(23.1)$ & $0(0.0)$ \\
\hline Hanging or strangulation & - & $6(5.2)$ & $2(1.8)$ \\
\hline Stabbing & - & $1(0.9)$ & $0(0.0)$ \\
\hline Self-biting & - & $0(0.0)$ & $1(0.9)$ \\
\hline Self-hitting & - & $0(0.0)$ & $1(0.9)$ \\
\hline History of suicidal behavior & 64 (84.3) & $96(82.1)$ & $92(82.9)$ \\
\hline History of ED visit ${ }^{\dagger \ddagger}$ & $54(71.1)$ & 46 (39.3) & $48(43.2)$ \\
\hline History of psychiatric treatment & $65(85.5)$ & $92(78.6)$ & $85(76.6)$ \\
\hline History of psychiatric admission ${ }^{\ddagger \ddagger}$ & $56(73.7)$ & 46 (39.3) & $47(42.3)$ \\
\hline \multicolumn{4}{|l|}{ Child abuse history } \\
\hline No & $53(69.7)$ & $47(40.2)$ & $49(44.1)$ \\
\hline Yes ${ }^{*+\neq}$ & $16(21.1)$ & $56(47.8)$ & $53(47.8)$ \\
\hline Sexual & $0(0.0)$ & $13(11.1)$ & $4(3.6)$ \\
\hline Physical & $6(7.9)$ & $14(12.0)$ & $12(10.8)$ \\
\hline Emotional $^{\dagger \ddagger}$ & $12(15.8)$ & $44(37.6)$ & $44(39.6)$ \\
\hline Neglect $t^{\dagger \ddagger}$ & $7(9.2)$ & $29(24.8)$ & $27(24.3)$ \\
\hline Unknown & $7(9.2)$ & $14(12.0)$ & $9(8.1)$ \\
\hline \multicolumn{4}{|l|}{ Familial psychiatric history } \\
\hline No & $40(52.6)$ & $54(46.2)$ & $43(38.7)$ \\
\hline Yes & $26(34.2)$ & $28(23.9)$ & $40(36.0)$ \\
\hline Unknown & $9(11.8)$ & $35(29.9)$ & $28(25.2)$ \\
\hline \multicolumn{4}{|l|}{ Major stressor* } \\
\hline Relationship with family ${ }^{\ddagger}$ & $7(9.2)$ & 46 (39.3) & $47(42.3)$ \\
\hline Peer relationship & $9(11.8)$ & $23(19.7)$ & $16(14.4)$ \\
\hline Romantic relationship & $7(9.2)$ & $7(6.0)$ & 4 (3.6) \\
\hline Academic stress & $9(11.8)$ & $21(18.0)$ & $8(7.2)$ \\
\hline School adaptation & $3(3.9)$ & $8(6.8)$ & $5(4.5)$ \\
\hline School violence & $1(1.3)$ & $0(0.0)$ & $0(0.0)$ \\
\hline No specific stressor ${ }^{\ddagger \ddagger}$ & $46(60.5)$ & $32(27.3)$ & $39(35.1)$ \\
\hline \multicolumn{4}{|l|}{ Psychiatric diagnosis* } \\
\hline Depression $^{\dagger \ddagger}$ & $36(47.4)$ & $86(73.5)$ & $81(73.0)$ \\
\hline Adjustment disorder & $7(9.2)$ & $15(12.8)$ & $11(9.9)$ \\
\hline Bipolar disorder $^{\ddagger \ddagger}$ & $22(28.9)$ & $11(9.4)$ & $8(7.2)$ \\
\hline PTSD & $2(2.6)$ & $11(9.4)$ & $3(2.7)$ \\
\hline Anxiety disorder & $3(3.9)$ & $3(2.6)$ & $3(2.7)$ \\
\hline Somatic symptom disorder & $1(1.3)$ & $2(1.7)$ & $2(1.8)$ \\
\hline Psychotic disorder & $3(3.9)$ & $2(1.7)$ & $0(0.0)$ \\
\hline OCD & $2(2.6)$ & $2(1.7)$ & $4(3.6)$ \\
\hline Eating disorder & $1(1.3)$ & $1(0.9)$ & $0(0.0)$ \\
\hline \multicolumn{4}{|l|}{ Disposition of ED } \\
\hline Admission to psychiatry ${ }^{\ddagger \S}$ & $16(21.1)$ & $27(23.1)$ & $11(9.9)$ \\
\hline Admission to other departments or transfer ${ }^{\dagger \S}$ & $0(0.0)$ & $11(9.4)$ & $3(2.7)$ \\
\hline Discharge with follow-up OPD visit ${ }^{\dagger \S}$ & $49(64.5)$ & $47(40.2)$ & $54(48.6)$ \\
\hline Discharge without follow-up OPD visit ${ }^{\dagger \neq \S}$ & $11(14.5)$ & $31(26.5)$ & $43(38.7)$ \\
\hline Death & $0(0.0)$ & $1(0.9)$ & $0(0.0)$ \\
\hline
\end{tabular}

ED, emergency department; SD, standard deviation; PTSD, post-traumatic stress disorder; OCD, obsessive-compulsive disorder; OPD, outpatient department. Values are presented as number (\%).

${ }^{*}$ Multiple choices were allowed, ${ }^{\dagger} p<0.05$ for suicidal ideation only and suicidal self-harm, ${ }^{\ddagger} p<0.05$ for suicidal ideation only and non-suicidal self-harm, ${ }^{\S} p<0.05$ for suicidal self-harm and non-suicidal self-harm. 
Comparison between single and multiple visit groups Approximately $24.4 \%$ ( $\mathrm{n}=41$; female 35 , male 6 ) of patients had one or more self-harm attempts after the index episode, and

Table 3. Comparison of Characteristics between Patients with a Single Visit and Those with Multiple Visits to the ED for Self-Harm

\begin{tabular}{|c|c|c|}
\hline & $\begin{array}{l}\text { Single visit } \\
(n=127)\end{array}$ & $\begin{array}{l}\text { Multiple visits } \\
\text { (n=41) }\end{array}$ \\
\hline Number of episodes & 127 & 177 \\
\hline Sex, female & $102 / 127(80.3)$ & $35 / 41(85.3)$ \\
\hline Age (yr), mean $\pm S D$ & $15.94 \pm 1.72$ & $15.92 \pm 1.68$ \\
\hline $12-15$ & $50(39.3)$ & $14(34.1)$ \\
\hline $16-18$ & $77(60.7)$ & $27(65.9)$ \\
\hline $\begin{array}{l}\text { Repetition interval between visits (days) } \\
\text { (median, range) }\end{array}$ & - & $30(1-602)$ \\
\hline History of psychiatric treatment ${ }^{\dagger}$ & $74(58.3)$ & $35(85.4)$ \\
\hline History of psychiatric admission ${ }^{\dagger}$ & $18(14.2)$ & $18(43.9)$ \\
\hline \multicolumn{3}{|l|}{ Child abuse history $^{\dagger}$} \\
\hline No & $62(48.8)$ & $16(39.0)$ \\
\hline Yes $^{* \dagger}$ & $41(32.3)$ & $22(53.7)$ \\
\hline Sexual $^{\dagger}$ & $4(3.1)$ & $5(12.2)$ \\
\hline Physical & $17(13.4)$ & $4(9.8)$ \\
\hline Emotional $^{\dagger}$ & $32(25.2)$ & $18(43.9)$ \\
\hline Neglect & $23(18.1)$ & $12(29.3)$ \\
\hline Unknown & $24(18.9)$ & $3(7.3)$ \\
\hline \multicolumn{3}{|l|}{ Familial psychiatric history ${ }^{\dagger}$} \\
\hline No & $58(45.7)$ & $20(48.8)$ \\
\hline Yes $^{\dagger}$ & $17(13.4)$ & $13(31.7)$ \\
\hline Unknown ${ }^{\dagger}$ & $52(40.9)$ & $8(19.5)$ \\
\hline Alcohol consumption $^{\dagger}$ & $11 / 127(8.7)$ & $2 / 177(1.1)$ \\
\hline \multicolumn{3}{|l|}{ Reason for visits } \\
\hline Suicidal ideation only ${ }^{\dagger}$ & $18 / 127(14.2)$ & $58 / 177(32.8)$ \\
\hline Suicidal self-harm & $56 / 127(44.1)$ & $61 / 177(34.5)$ \\
\hline Non-suicidal self-harm & $53 / 127(41.7)$ & $58 / 177(32.8)$ \\
\hline \multicolumn{3}{|l|}{ Key method } \\
\hline Self-poisoning & $54(42.5)$ & $16(39.0)$ \\
\hline Self-cutting & $36(28.3)$ & $9(22.0)$ \\
\hline Charcoal burning & $3(2.4)$ & $0(0.0)$ \\
\hline Jumping from the height ${ }^{\dagger}$ & $13(10.2)$ & $10(24.4)$ \\
\hline Hanging or strangulation ${ }^{\dagger}$ & $1(0.8)$ & $4(9.8)$ \\
\hline Ideation only ${ }^{\dagger}$ & $18(14.2)$ & $2(4.9)$ \\
\hline Others & $2(1.6)$ & $0(0.0)$ \\
\hline \multicolumn{3}{|l|}{ Disposition of ED } \\
\hline Admission to psychiatry & $17 / 127(13.4)$ & $37 / 177(20.9)$ \\
\hline $\begin{array}{l}\text { Admission to other departments or } \\
\text { transfer }^{\dagger}\end{array}$ & 10/127 (7.9) & 4/177 (2.3) \\
\hline Discharge with follow up OPD visit ${ }^{\dagger}$ & $31 / 127(24.4)$ & 119/177 (67.2) \\
\hline Discharge without follow up OPD visit ${ }^{\dagger}$ & $69 / 127(54.3)$ & 16/177 (9.0) \\
\hline Death & $0 / 127(0.0)$ & 1/177 (0.6) \\
\hline
\end{tabular}

ED, emergency department; OPD, outpatient department.

Values are presented as number (\%). Reason for visit and ED results were calculated using the number of ED visit episodes as the denominator, and the number of patients as the denominator otherwise.

${ }^{*}$ Multiple choices were allowed, ${ }^{\dagger} p<0.05$ comparison between the two groups.
177 self-harm episodes were recorded in the multiple visit group (Table 3). Among the 41 patients who repeatedly inflicted self-harm, 22 (53.7\%) patients repeated once, 6 (14.6\%) twice, $4(9.8 \%)$ thrice, and $9(21.9 \%)$ repeated 4 or more times during the study. The median interval of repetition between visits was 30 days (range 1-602 days). Among 117 episodes in multiple visits, repeated visits after over 6 months were 11 episodes, and 2 episodes re-visited the ED after over 1 year. There was no statistical difference in sex and age between the two groups. The multiple visit group tended to have more history of psychiatric treatment/admission ( $58.3 \%$ vs. $85.4 \%, p=0.002 ; 14.2 \%$ vs. $43.9 \%$, $p<0.001$ ), history of child abuse ( $32.3 \%$ vs. $53.7 \%, p=0.013$ ), and familial psychiatric history ( $13.4 \%$ vs. $31.7 \%, p=0.008)$ compared to single visit group. Among the history of child abuse, sexual and emotional types of child abuse were more common in the multiple visit group ( $3.1 \%$ vs. $12.2 \%, p=0.040 ; 25.2 \%$ vs. $43.9 \%, p=0.023)$. However, an unknown history of familial psychiatric history was more common in the single visit group ( $40.9 \%$ vs.19.5\%; $p=0.012$ ), probably due to limited interview at ED. Concerning the reasons for visit to $\mathrm{ED}$, episodes with suicidal ideation only were more common in the multiple visit group than in the single visit group ( $14.2 \%$ vs. $32.8 \%, p<0.001)$, but episodes with suicidal attempt or non-suicidal self-harm episodes were not different. In the key methods of the multiple visit group, jumping from height ( $10.2 \%$ vs. $24.4 \%, p=0.021$ ) and hanging/strangulation ( $0.8 \%$ vs. $9.8 \%, p=0.013)$, which were generally considered as more lethal methods, were significantly higher. Self-poisoning and self-cutting were not significantly different ( $p=0.720$ and $p=0.544$ ). In contrast, those who did not choose any method other than complaining about suicidal ideation were only two, and they were not statistically significant ( $14.2 \%$ vs. $4.9 \%, p=0.164$ ). While discharge from ED with follow-up OPD visit was higher in the multiple visits group ( $p<$ 0.001 ), admission to another department or transfer and discharge without follow-up OPD visit was higher in the single visit group ( $p=0.027$ and $p<0.001$, respectively). Admission to psychiatry was not different between the two groups $(p=0.091)$.

\section{DISCUSSION}

We have investigated a consecutive series of children and adolescents aged 12 to 18 years who presented to the ED following self-harm between 2015 and 2019. During the study, the number of patients and episodes with self-harm increased, and were higher among female than in male patients. When comparing groups based on the reason for the visit, there were differences in the methods used for self-harm, major stressor, and underlying psychiatric diagnosis. Additionally, repeated ED visitors with self-harm had more history of psychiatric treatment/admission, history of child abuse, familial psychiatric history compared to single visit patients. Key methods among multiple ED visits also tended to be more lethal, such 
as jumping from height and hanging/strangulation.

Recent studies indicated an increasing trend of self-harm in adolescents worldwide. In the present study, "self-harm" referred to both suicidal attempt and non-suicidal self-injury, ${ }^{16}$ with injurious behavior or with ideation only. In one meta-analysis of community-based studies, ${ }^{17}$ the prevalence of self-harm in adolescents increased between 1990 and 2015, showing a lifetime prevalence of $16.9 \%$. In particular, based on the latest data up to 2019 from this study, there has been a steep increase in the number of ED visits for self-harm since 2018. The reason for this increase is still unclear, but one possible explanation may include the rapid increase in self-harm-related content on the internet, based on social media. Among young people, social networking and picture/video sharing platforms, such as Instagram and YouTube, have become increasingly popular in recent years. Social network services have also received considerable attention in Korea due to the influence of selfharm contents uploaded by celebrities around the year 2018, as well as a widespread phenomenon of so-called "self-harm certification" in which adolescents upload pictures of selfharm with a hashtag of "self-harmer" using their "self-harm accounts." ${ }^{18}$ Due to growing concerns about hazardous contents on the internet, the Korean Ministry of Health and Welfare has been constantly performing "self-harm and suicide contents cleaning activity." During the 2 weeks of intensive monitoring in July 2018, it was found that the number of pictures or videos about self-harm and suicide reported on Korean websites had increased by $3728 \%$ ( $n=8039$ ), compared to the same period in $2017(\mathrm{n}=210)$, and self-harm certifying pictures were most common (6808 cases, $84 \%) .{ }^{19}$ It is known that contact with others engaging in self-harm is one of the major risk factors for self-harm. ${ }^{1}$ Although these factors related to social media may help to provide a sense of community and encouragement for recovery, it is presumed that vulnerable adolescents may be easily triggered to show imitational behaviors, due to their exposure to self-harm related contents. ${ }^{20} \mathrm{~A}$ systematic review found that internet use may exert a negative influence on adolescents by normalizing self-harm and potentially discouraging them from seeking professional help. The internet can also be used for "cyber-bullying" of peers, leading to a relationship with increased risk of self-harm. ${ }^{21}$ A more recent study reported that exposure to self-harm on Instagram was related to self-harm and suicidality-related outcomes. ${ }^{22}$ As our results have revealed the rapidly increasing trend of ED visits for selfharm among Korean adolescents, further study is required to confirm the relationship between this trend and the exposure to self-harm contents on social media.

In line with previous studies, ${ }^{23,24}$ self-cutting was the leading method of self-harm in the non-suicidal group, in contrast to the more lethal methods, while jumping from height was higher in the suicidal group. Interestingly, in both the suicidal and non-suicidal self-harm groups, a significant proportion of substance used in self-poisoning were drugs that were prescribed by doctors (Supplementary Table 2, only online); therefore, physicians should be careful to ensure that adolescents are monitored with regard to their compliance in taking the medicines prescribed to them appropriately.

The increase in the number of episodes was more than the increase in the number of people, which is attributed to the increase in the number of multiple visit patients. Since the repetition of deliberate self-harm is known to increase the risk of further self-harm and eventual suicide,,$^{12,25,26}$ it is important to understand the factors associated with multiple ED visits. In this study, about $24.4 \%$ of the patients repeatedly visited ED with self-harm after an index episode, which was much higher than the proportion reported in previous studies, ${ }^{15,27}$ probably due to different clinical settings. The higher proportion of prior psychiatric treatment and psychiatric admission, familial psychiatric history, and child abuse history in multiple ED visits were in accordance with the results of previous studies, ${ }^{1,28,29}$ which suggest that these are important risk factors for the repetition of self-harm and suicide in adolescents. In one study, ${ }^{28}$ the severity of symptoms and distance to the hospital were also suggested to be the risk factors for repeated ED visits; however, these factors could not be investigated due to the lack of information from ED. Moreover, despite the importance of this information in predicting repetition, the history of child abuse and familial psychiatric treatment was not obtained properly in a considerable proportion of the ED visit cases. In addition, a structured form to collect information for self-harm in the ED might help to select the high-risk adolescents for repetition of self-harm and individualize the intervention strategy.

Interestingly, the proportion of alcohol consumption was higher in the single visit group than in the multiple visit group. Acute alcohol intoxication is a known risk factor for suicidal behavior, and it also increases psychological distress, aggressiveness, suicide-specific alcohol expectancies, and cognitive constriction, which impairs the generation and implementation of alternative coping strategies. ${ }^{30}$ Non-suicidal self-injury also co-occurs with problematic alcohol use, and a higher risk of suicide has been associated with a potentially harmful combination of behaviors. Among personality traits, impulsivity has been independently linked to both self-harm and alcohol use and may, therefore, represent a potential underlying behavioral mechanism. ${ }^{31}$ In one systematic review, ${ }^{32}$ mood-based impulsivity was associated with the initiation of self-harm, while cognitive facets of impulsivity were related to the maintenance of self-harm. To explain the different proportions of alcohol consumption between groups, further study is required to determine which facets of trait impulsivity are elevated in the individuals who visit the ED single or multiple times for either self-harm or problematic alcohol consumption.

Although the number of monthly episodes decreased in March and August, which are the months when the spring and fall semesters begin, the difference was not statistically significant, mainly due to the limited number of ED visit episodes by 
the months. Moreover, academic stress or school adaptation was not the leading stressor in all groups. The result did not correspond with the previous study based on postmortem reports of suicides, whichh reported that for middle and high school students in Korea, the death toll from suicides increased during the school term, especially in March, and decreased during vacation periods. ${ }^{33}$ Although this discrepancy might be attributed to the different study settings, the monthly or seasonal variation should be further analyzed with nationwide ED data to identify the relationship between the academic calendar and ED visits for self-harm.

This study had several limitations. First, the patients in this study were from an ED of a single hospital with a relatively small population; therefore, our results cannot be generalized to all Korean adolescents presenting to clinical services and cannot represent the community population. However, presenting to the ED itself can be a risk factor associated with suicidal thoughts and behaviors, and it is known that ED is the most common setting for adolescents who sustain clinically serious self-harm. ${ }^{8}$ Therefore, it is meaningful to understand the clinical characteristics of adolescents who visit $\mathrm{ED}$, as well as the factors associated with multiple visits and the repetition of selfharm. Second, due to the retrospective nature of this study with ED medical records, information may be scarce and incomplete, particularly in single visit cases. For example, the reason for the different proportions of depression and bipolar disorder between groups was not clear in this study. Besides, due to the lack of limited quantitative data, such as the self-injury trauma scale, it was not possible to classify the identified cases in terms of severity. However, board-certified psychiatrists with experiences of child and adolescent psychiatry thoroughly reviewed not only the ED psychiatric consultation notes, but also the medical records of outpatient clinic and admission after ED visit, to gather in-depth information. Third, since only one patient died of suicidal attempt at the ED despite urgent treatment after jumping from a high place, not all deaths from suicide may have been included in our study. Since it is difficult to confirm the cause of death at the $\mathrm{ED}$, the code for a patient who was dead on arrival was likely to be inserted as "illdefined and unknown cause of mortality (R99)" instead of a code indicating suicidal intent, such as "intentional self-harm by hanging." In the present study, the cases with R99 were not included, as the intent of self-harm could not be distinguished only with ED profiles in a significant portion of the cases.

In conclusion, we identified the increasing trend of ED visits and the increase of repeated ED visits for self-harm in Korean adolescents. For self-harm adolescents, the history of psychiatric treatment/admission, child abuse, and familial psychiatric history should be properly obtained to identify the risk for multiple ED visits and future suicidal attempts. To prevent self-harm in adolescents, further studies are needed to explore the association between this upsurge flow of self-harm and increasing self-harm contents on the internet, and ascer- tain the risk factors of multiple ED visits for appropriate afterED visit care plans.

\section{AUTHOR CONTRIBUTIONS}

Conceptualization: Jooah Cheon and Keun-Ah Cheon. Data curation: Jooah Cheon. Formal analysis: Donghun Oh. Investigation: Junghan Lee and Jaeun Ahn. Methodology: Keun-Ah Cheon. Validation: Jooah Cheon and Keun-Ah Cheon. Project administration: Jooah Cheon and Keun-Ah Cheon. Resources: Donghun Oh. Software: Junghan Lee. Supervision: Dong-Ho Song. Visualization: Jaeun Ahn. Writingoriginal draft: Jooah Cheon. Writing_review \& editing: Keun-Ah Cheon. Approval of final manuscript: all authors.

\section{ORCID iDs}

Jooah Cheon

Donghun Oh

Junghan Lee

Jaeun Ahn

Dong-Ho Song
Keun-Ah Cheon https://orcid.org/0000-0001-8399-2057 https://orcid.org/0000-0003-3824-4783 https://orcid.org/0000-0002-2367-867X https://orcid.org/0000-0002-1331-7854 https://orcid.org/0000-0002-9647-3130 https://orcid.org/0000-0001-7113-9286

\section{REFERENCES}

1. Hawton K, Saunders KE, O'Connor RC. Self-harm and suicide in adolescents. Lancet 2012;379:2373-82.

2. Im Y, Oh WO, Suk M. Risk factors for suicide ideation among adolescents: five-year national data analysis. Arch Psychiatr Nurs 2017; 31:282-6.

3. Carroll R, Metcalfe C, Gunnell D. Hospital presenting self-harm and risk of fatal and non-fatal repetition: systematic review and meta-analysis. PLoS One 2014;9:e89944.

4. Kidger J, Heron J, Lewis G, Evans J, Gunnell D. Adolescent selfharm and suicidal thoughts in the ALSPAC cohort: a self-report survey in England. BMC Psychiatry 2012;12:69.

5. Insel BJ, Gould MS. Impact of modeling on adolescent suicidal behavior. Psychiatr Clin North Am 2008;31:293-316.

6. Jarvi S, Jackson B, Swenson L, Crawford H. The impact of social contagion on non-suicidal self-injury: a review of the literature. Arch Suicide Res 2013;17:1-19.

7. Zhu L, Westers NJ, Horton SE, King JD, Diederich A, Stewart SM, et al. Frequency of exposure to and engagement in nonsuicidal self-injury among inpatient adolescents. Arch Suicide Res 2016; 20:580-90.

8. Office of the Surgeon General, National Action Alliance for Suicide Prevention. Publications and Reports of the Surgeon General. 2012 national strategy for suicide prevention: goals and objectives for action. A Report of the U.S. Surgeon General and of the National Action Alliance for Suicide Prevention. Washington, DC: US Department of Health \& Human Services; 2012.

9. Cutler GJ, Flood A, Dreyfus J, Ortega HW, Kharbanda AB. Emergency department visits for self-inflicted injuries in adolescents. Pediatrics 2015;136:28-34.

10. Gilbody S, House A, Owens D. The early repetition of deliberate self harm. J R Coll Physicians Lond 1997;31:171-2.

11. Gunnell D, Bennewith O, Peters TJ, Stocks N, Sharp DJ. Do patients who self-harm consult their general practitioner soon after hospital discharge? A cohort study. Soc Psychiatry Psychiatr Epidemiol 2002;37:599-602.

12. Zahl DL, Hawton K. Repetition of deliberate self-harm and subse- 
quent suicide risk: long-term follow-up study of 11,583 patients. Br J Psychiatry 2004;185:70-5.

13. Sinclair JM, Gray A, Rivero-Arias O, Saunders KE, Hawton K. Healthcare and social services resource use and costs of self-harm patients. Soc Psychiatry Psychiatr Epidemiol 2011;46:263-71.

14. Larkin C, Di Blasi Z, Arensman E. Risk factors for repetition of selfharm: a systematic review of prospective hospital-based studies. PLoS One 2014;9:e84282.

15. Kwok CL, Yip PS, Gunnell D, Kuo CJ, Chen YY. Non-fatal repetition of self-harm in Taipei City, Taiwan: cohort study. Br J Psychiatry 2014;204:376-82.

16. Kapur N, Cooper J, O'Connor RC, Hawton K. Non-suicidal selfinjury v. attempted suicide: new diagnosis or false dichotomy? $\mathrm{Br}$ J Psychiatry 2013;202:326-8.

17. Gillies D, Christou MA, Dixon AC, Featherston OJ, Rapti I, GarciaAnguita A, et al. Prevalence and characteristics of self-harm in adolescents: meta-analyses of community-based studies 19902015. J Am Acad Child Adolesc Psychiatry 2018;57:733-41.

18. Seo M, Kim E, Lee T, Kim JH. Study on the development of an intervention model for high-risk youth: suicide and self-injury (Korean). Busan, South Korea: Korea Youth Counseling \& Welfare Institute (Ministry of Gender Equality and Family); 2018.

19. Ministry of Health and Welfare KSPC. Self-harm and suicide contents cleaning activity (Korean) [accessed on 2019 December 20]. Available at: https://www.gov.kr/portal/ntnadmNews/1574871.

20. Brown RC, Fischer T, Goldwich AD, Keller F, Young R, Plener PL. \#cutting: non-suicidal self-injury (NSSI) on instagram. Psychol Med 2018;48:337-46.

21. Daine K, Hawton K, Singaravelu V, Stewart A, Simkin S, Montgomery $\mathrm{P}$. The power of the web: a systematic review of studies of the influence of the internet on self-harm and suicide in young people. PLoS One 2013;8:e77555.

22. Arendt F, Scherr S, Romer D. Effects of exposure to self-harm on social media: evidence from a two-wave panel study among young adults. New Media Soc 2019;21:2422-42.
23. Carroll R, Thomas KH, Bramley K, Williams S, Griffin L, Potokar J, et al. Self-cutting and risk of subsequent suicide. J Affect Disord 2016;192:8-10.

24. Cully G, Corcoran P, Leahy D, Griffin E, Dillon C, Cassidy E, et al. Method of self-harm and risk of self-harm repetition: findings from a national self-harm registry. J Affect Disord 2019;246:843-50.

25. Owens D, Dennis M, Read S, Davis N. Outcome of deliberate selfpoisoning. An examination of risk factors for repetition. Br J Psychiatry 1994;165:797-801.

26. Tejedor MC, Díaz A, Castillón JJ, Pericay JM. Attempted suicide: repetition and survival--findings of a follow-up study. Acta Psychiatr Scand 1999;100:205-11.

27. Griffin E, McMahon E, McNicholas F, Corcoran P, Perry IJ, Arensman E. Increasing rates of self-harm among children, adolescents and young adults: a 10-year national registry study 2007-2016. Soc Psychiatry Psychiatr Epidemiol 2018;53:663-71.

28. Leon SL, Polihronis C, Cloutier P, Zemek R, Newton AS, Gray C, et al. Family factors and repeat pediatric emergency department visits for mental health: A Retrospective Cohort Study. J Can Acad Child Adolesc Psychiatry 2019;28:9-20.

29. Hawton K, Bergen H, Kapur N, Cooper J, Steeg S, Ness J, et al. Repetition of self-harm and suicide following self-harm in children and adolescents: findings from the Multicentre Study of Selfharm in England. J Child Psychol Psychiatry 2012;53:1212-9.

30. Hufford MR. Alcohol and suicidal behavior. Clin Psychol Rev 2001;21:797-811.

31. Hasking P. Differentiating non-suicidal self-injury and risky drinking: a role for outcome expectancies and self-efficacy beliefs. Prev Sci 2017;18:694-703.

32. Lockwood J, Daley D, Townsend E, Sayal K. Impulsivity and selfharm in adolescence: a systematic review. Eur Child Adolesc Psychiatry 2017;26:387-402.

33. Lee D, Jung S, Park S, Lee K, Kweon YS, Lee EJ, et al. Youth suicide in Korea across the educational stages. Crisis 2020;41:187-95. 